\title{
Study on Contact Fatigue Life Prediction for Clasp Joint Structure of Mooring Buoy
}

\author{
Zifan Fang ${ }^{\text {a,* }}$, Daojia Du ${ }^{\text {a }}$, Kongde He ${ }^{\text {a }}$, Wenhui Shu ${ }^{\text {a }}$, Qingsong He ${ }^{\text {a }}$, Binfei Xiang ${ }^{\text {a }}$, \\ Huapan Xiao ${ }^{\mathrm{a}}$
}

${ }^{a}$ Hubei Key Laboratory of Hydroelectric Machinery Design \& Maintenance, China Three Gorges University, Yichang, Hubei Province, China

\begin{abstract}
This paper focuses on predicting the contact fatigue life of the mooring buoy clasp joint structure by using the finite element program ANSYS/Fatigue. Mises equivalent stress is choosed as a stress-related fatigue criterion. An evaluation method of fatigue life under the pressure of buoyancy and wave force is established for the mooring buoy clasp joint structure. Firstly, wave force is treated as Morison dynamic force here and mooring buoys vibration model is conducted to analyze the dynamic response characteristics of vertical vibration of the mooring buoy. Secondly, dynamic contact stress calculations are conducted by directly exerting calculated vibration loads on the mooring buoy, and the stress spectrums of nodes are obtained. Finally, the S-N material fatigue curve and the Miner linear cumulative damnification principle are commonly applied to evaluate the contact fatigue life of the mooring buoy. The results show that the finite element program ANSYS/Fatigue can solve the contact fatigue problem simply, economically and efficiently. It also provides an effective engineering analysis method for these kinds of fatigue problems of the underwater mooring buoy.
\end{abstract}

Index Terms: mooring buoys; multi-body contact; wave force; fatigue life

(C) 2012 Published by MECS Publisher. Selection and/or peer review under responsibility of the Research Association of Modern Education and Computer Science.

\section{Introduction}

Underwater mooring buoy is a new, long-term, fixed, and unmanned monitoring system for underwater environmental factor, which has broad application prospects in monitoring dams or ports, detecting damage of the underwater constructions and data acquisition of underwater environment. Mooring buoy is composed of many separate sealed floats that are jointed together by metal clasps. The clasp joint structure is installed into the pipe groove, and the gradient cooperated with the nut has self-locking function, so that clasp joint

\footnotetext{
* Corresponding author.

E-mail address: fzf@ctgu.edu.cn
} 
structure which has a high bonding strength is used in underwater mooring and also easy to process, install and maintain. The final engineering practicality will be affected directly structural performance. Therefore, it is necessary to calculate and analyze the ontology structure [1] of mooring buoy, and then study the properties such as stress distribution at the clasp joint point, and so on, which would be compared with technical indicators.

Clasp joint structure is a typical surface-surface contact conjunction from the perspective of the mechanical model. The contact problem involves stress concentration, which is an uncertain boundary problem and a highly nonlinear behavior. It is difficult to get the real force and deformation by using common methods. The nonlinear finite element theory [2] is applied to analyze the contact force of the clasp joint structure. It provides a basis for further improving of the clasp joint structure. Meanwhile, the underwater mooring buoy floats in the water. It will vibrate under the action of wave force, which may lead to fatigue failure [3] of the clasp joints structure. Morison equation can describe the wave force of mooring buoy accurately. Based on the contact analysis, a vibration model of mooring buoy is established according to the Morison equation $[4,5]$, and is used to analyze the vibration property. Finally, the fatigue life of the clasp joint structure in accordance with Miner linear cumulative fatigue damage theory $[6,7,8]$ is predicted.

\section{Finite Element Contact Model of Clasp Joint Structure}

Clasp joint structure of mooring buoy is shown in Fig. 1. The mooring buoy is composed of buoys, bolts, clasps, and connectors. Four pairs of bolts surround the clasps uniformly. Two adjacent buoys are connected by the clasps and bolts. It is an important step to design appropriate clasp joint structure and check its strength and rigidity by finite element software.

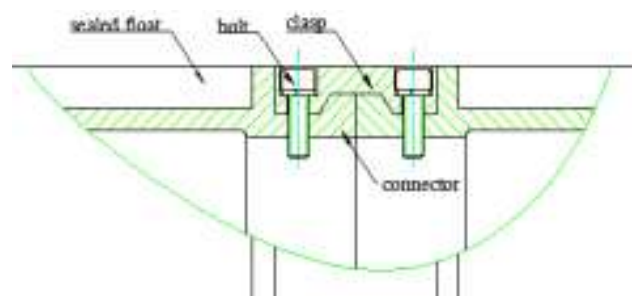

Figure. 1. The diagram of clasp joint structure of mooring buoy

\subsection{Finite element nonlinearity methods for solving the multi-body contact problem}

The deformation tendency of clasp joint structure under the buoyancy can be judged by force analysis. The bending moment would be formed on the local of buoy, which leads to eversion phenomenon of outer-ring edge. The clasp will absorb bending moment result from the contact problem between buoy and clasp as well. From the mechanical perspective, these problems belong to contact nonlinear problem. It is difficult to calculate the stress and strain using the traditional elastic contact algorithm. In recent years, with the continuous improvement of nonlinear theory and rapid development of computer technology, the nonlinear finite element method that is used to analyze these issues has become increasingly mature.

1) Contact boundary conditions

The mooring buoy clasp, buoy, bolts and screws, these four objects contact with each other under the buoyancy and external disturbance. The stiffness equation could be:

$$
K^{\alpha} \delta^{\alpha}=P^{\alpha}
$$

Where $K^{\alpha}$ is the stiffness matrix of the $\alpha$ th object, $\delta^{\alpha}$ is the nodal displacements matrix of the $\alpha$ th object, and $P^{\alpha}$ is the nodal forces matrix of the $\alpha$ th object. 
The object boundary condition is given by

$$
\Gamma^{\alpha}=\Gamma_{p}^{\alpha}+\Gamma_{n}^{\alpha}
$$

Where $\Gamma_{p}^{\alpha}$ is the potential contact boundary under loading, $\Gamma_{n}^{\alpha}$ is the impossible contact boundary under loading.

As we know, $\Gamma_{p}^{\alpha}$ is variable under dynamic loading. $\Gamma_{p}^{\alpha}$ is given by

$$
\Gamma_{p}^{\alpha}=\Gamma_{c}^{\alpha}+\Gamma_{f}^{\alpha}
$$

In (1), it is easy to find that only if we know either the nodal force components or nodal displacement components, can the other unknown quantity be solved. However, the nodal force components and nodal displacement components are all unknown on the contact boundary $\Gamma_{c}^{\alpha}$. This is a nonlinear problem of boundary constraint, which should be solved only in iterative method and complementary method.

To solve (1), the connection status of contact surface must be regarded as the definite condition of complementary equation. On the contact boundary $\Gamma_{c}^{\alpha}$, the equilibrium condition and deformation compatibility condition between the contact bodies A and B are defined as

Equilibrium condition $\quad U_{i}^{A}=U_{i}^{B}$

Compatibility condition $\boldsymbol{u}_{i}^{A}+\boldsymbol{u}_{i}^{B}=\Delta_{i}^{O}$

Where $U_{i}^{A}$ and $U^{B}{ }_{i}$ are normal nodal force of node $\mathrm{i}$ of the contact bodies $A$ and $B$, respectively. $U^{A}{ }_{i}$ and $U^{B}{ }_{i}$ are normal nodal displacement of node $i$ on the contact bodies $A$ and $B$, respectively. $\Delta^{O}{ }_{i}$ is the initial clearance between $i_{A}$ and $i_{B}$.

\section{2) Boundary convergence criterion}

Since the finite element method is a typical structural discrete method, the number of discrete nodes has an impact on analysis accuracy. Therefore, the engineering problems usually provide an allowed tolerance in dealing with contact problems. The allowed tolerance is given by

$$
\left|\left(u_{i}^{\alpha}\right)_{j}-\left(u_{i}^{\alpha}\right)_{j-1}\right| \leq 1
$$

If the numerical simulation results satisfy (6), the boundary is convergent. Otherwise, the mesh in the junction of $\Gamma_{c}^{\alpha}$ and $\Gamma_{f}^{\alpha}$ must be refined, and the numerical simulation also need to be carried out again until the simulation results satisfy (6), where $\mathrm{j}$ is the iteration number.

\subsection{Establishing contact model of clasp joint structure}

Contact problem is a very common nonlinear behavior, which is a special and important subset in nonlinear type of state change. Finite element analysis software ANSYS supports three contact styles: point-point contact, point-surface contact, surface-surface contact. Finite element contact model of clasp joint structure is a surface-surface contact problem and supports normal load on contact surface. Finite element contact model of clasp joint structure applies the surface-surface contact algorithm. Multiple contact pairs should be defined considering the contact relationship among clasp, buoys and screw bolts.

The contact pairs use Coulomb's friction law. The interface friction coefficient is 0.1 . The finite element software ANSYS usually uses Lagrange Algorithm to solve the contact friction problem. In order to ensure the efficiency and stability, automatic time step and linear search techniques are used in calculation.

\section{The Fatigue Life Analysis of Mooring Buoy under Wave Force}

First of all, the vibration problem of mooring buoy should be simplified as a free vibration when dealing with vibration problem of mooring buoy under wave force. The following assumptions are made as: 1) 
Material of mooring buoys is linear elastic. 2) The mooring buoy is simplified as an extensional beam. 3) Vertical and horizontal vibration of the mooring buoy are not coupled.

The computational model is shown in Fig. 2. Establishing the $\mathrm{x}, \mathrm{y}, \mathrm{z}$ coordinates with the midpoint of suspended span as origin. The mooring buoy length is $\mathrm{L}$, the distance between buoy and the surface of water is $e$. The direction of wave force is perpendicular to the buoy axis. This paper only considers the vertical vibration of mooring buoy along direction y under the wave force.

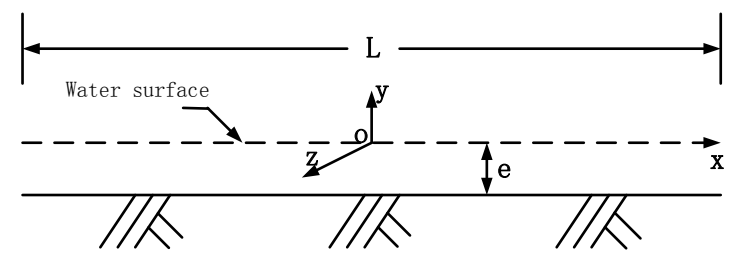

Figure. 2 Ideal model of mooring buoy

\subsection{Dynamic Response Analysis of vertical vibration of Mooring buoys}

Vertical vibration motion equation of mooring buoys is created based upon Morison hydrodynamic equation and the force action on the mooring buoy in the vertical direction. The equation is given by

$$
m_{s} \ddot{y}+\left(c_{s}+c_{v}\right) \dot{y}+k_{s} y=f_{L}
$$

Where $c_{s}$ and $c_{v}$ are buoy damping coefficient and seawater damping coefficient, respectively. $f_{L}$ is wave lift. And $y, \dot{y}$, and $\ddot{y}$ are vertical vibration displacement, vertical vibration velocity and vertical vibration acceleration of buoy, respectively.

\subsection{Fatigue life analysis of mooring buoy}

Mooring buoy fatigue failure under the flutter of wave lift is vertical vibration fatigue failure. The fatigue damage evaluation process is described as following four steps.

Step one: selecting the appropriate S-N curve.

S-N curve is defined as follows:

$$
N=C \cdot S^{-m}
$$

Where $\mathrm{N}$ is the cycle index of fatigue failure for a stress of $S$ that defined by S-N curve. $M$ is the fatigue index, $C$ is the characteristics constant of fatigue strength.

$\mathrm{S}-\mathrm{N}$ curve is usually obtained by the experiment, and the experimental conditions should be consistent with the above parameters. If the experimental conditions are different from fatigue conditions of mooring buoy, the reasonable corrective measures should be taken to amend it.

For each considered critical position, the appropriate S-N curve is selected based on geometric properties, loads, material properties, welding method, welding quality, and so on.

Step Two: Calculating the cumulative damage

The total cycle number $n_{i}$ for each stress level that corresponding to each sea conditions in the whole usage cycle should be calculated firstly. For continua, the cycle index $N_{i}$ corresponding to each stress level is founded according to S-N curve. Consequently, the value $n_{i} / N_{i}$ caused by each stress level can be calculated based on $n_{i}$ and $N_{i}$. $\frac{\sum n_{i}}{N_{i}}$ for each node in one year can be obtained according to the Miner linear cumulative damnification principle.

Step Three: Calculating the fatigue life

The fatigue life of marine structure is given by 


$$
N=\frac{1}{\sum \frac{n_{i}}{N_{i}}}
$$

Step Four: Evaluation criterion of fatigue damage

The design philosophy of mooring buoys is that the vortex vibration caused by wave is allowed, but the stress cycle index should meet evaluation criterion of fatigue damage. Evaluation criterion of fatigue damage is based on Miner rule:

$$
D=\sum_{i=1}^{s} \frac{n_{i}}{N_{i}} \leq \eta
$$

Where $D$ is the cumulative fatigue damage life, $\eta$ is the cumulative fatigue damage ratio, $S$ is the stress difference.

\section{Examples and Analysis}

Mooring buoy is composed of many separate sealed floats that are jointed together by metal clasps. The shell thickness, materials and the specific size of clasps joint structure are omitted. The two pieces of metal heads and clasp are jointed together rigidly by bolts.

We start our study with static stress analysis by finite element software. Then, we carry out fatigue analysis based upon static stress analysis. The specific steps are shown in Fig. 3. FATIGUE module adopts the linear cumulative fatigue damage theory and widely used stress-life approach which considers every aspect fatigue factors such as the mean stress, load conditions, and fatigue strength coefficients for fatigue analysis.

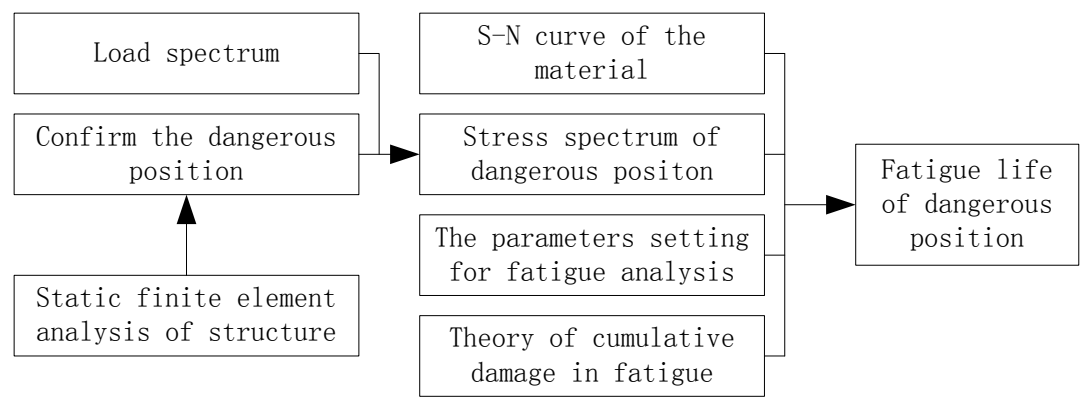

Figure. 3. The steps of fatigue analysis

\subsection{The stress intensity analysis}

The stress distribution of mooring buoy clasp joint structure under the action of buoyancy is shown in Fig. 4. As shown in Fig. 4, the stress at the ends of clasp is maximum. The maximum stress is $62.7 \mathrm{MPa}$ which does not exceed the material yield limit. Meanwhile, the stress distribution at the ends of clasp is similar. However, due to the contact between connector of mooring buoy and clasp, it results in stress concentration on the clasp. The similar stress distribution of mooring buoys clasp structure emerges under the combined action of buoyancy and wave lift. The maximum stress reaches up to $88.7 \mathrm{MPa}$. 


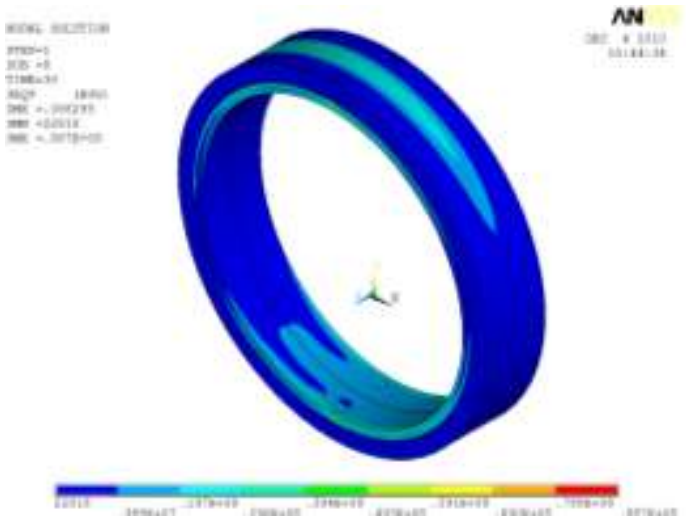

Figure. 4. The stress distribution of clasps joint structure under the wave lift

\subsection{Fatigue Analysis}

After the stress calculation, the fatigue analysis is conducted in the general post-processor POST1.The Mises equivalent stress contour of clasps joint structure is shown in Fig. 4. It shows that the stress is relatively concentrated on the connector of buoy and clasp. The maximum Mises equivalent stress emerges at the node 572 , which is the most dangerous point of fatigue failure. From Fig. 4, the Mises equivalent stress is $62.7 \mathrm{MPa}$ under the action of the buoyancy, meanwhile the Mises equivalent stress is $88.7 \mathrm{MPa}$ under the combined influence of buoyancy and wave lift. The stress value is calculated according to the third strength theory.

1) The determination of fatigue parameters

a) There are one node location and one event in fatigue calculation, and each event has two loads, which are the maximum load and minimum load, respectively.

b) The material fatigue property is defined by the S-N curve, which in fact shows the relationship between the stress amplitude (Smax-Smin)/2 and the fatigue cycle index.

2) Stress, cycle number of specified event and scale factor

a) Applying the FS command, the Mises stresses under minimum load and maximum load are stored artificially (not obtained from the result file Jobname.RST). Applying the FSNODE command, the Mises stresses that concluding six stress components are obtained from the result file Jobname.RST.

b) The period statistical characterization and wave height of sea areas in which mooring buoy is are defined as follows: The mean wave height $\bar{H}=5.13 \mathrm{~m}$. The relationship between wave height and median period is $H_{1 / 10}=0.076 \bar{T}^{2}$. In addition, the relationship between $H_{1 / 10}$ and $\bar{H}$ is ${ }_{H_{1 / 10}}=1.83 \bar{H}$. Applying the above relations and data, the period of wave can be calculated as $\bar{T}=11.11 \mathrm{~s}$. Then the load cycle index $N$ for dangerous node 572 can be calculated (The scale factor is taken as 1), and the cycle index in one year is $N=2838526$.

3) The results of fatigue analysis

The accumulated fatigue life depletion of clasps joint structure can be obtained by means of contact fatigue analysis. As seen from the analysis, the accumulated fatigue life depletion of clasps joint structure is $0.236544<1$ in one year, which indicates that the clasp joint structure satisfies the requirement of fatigue life. The stress cycle index and the corresponding accumulated fatigue life depletion with using years are shown in Table 1. As shown in Table 1, the accumulated fatigue life depletion is $0.946174<1$ within four years. When running 5 years, the accumulated fatigue life depletion is $1.1827183>1$. As a result, the accumulated fatigue life for the clasp joint structure is 4 years. 
Table.1.Fatigue Life Result

\begin{tabular}{|l|c|c|}
\hline $\begin{array}{c}\text { Run-Time } \\
\text { /Years }\end{array}$ & $\begin{array}{c}\text { Number of } \\
\text { load cycle/N }\end{array}$ & $\begin{array}{c}\text { Accumulated life } \\
\text { depletion coefficient }\end{array}$ \\
\hline 1 & 2838523 & 0.236544 \\
\hline 2 & 5677046 & 0.473087 \\
\hline 3 & 8515569 & 0.709631 \\
\hline 4 & 11354092 & 0.946174 \\
\hline 5 & 14192615 & 1.182718 \\
\hline
\end{tabular}

\section{Conclusion}

In this paper, Mises equivalent stress acquired by finite element contact analysis is choosed as a stressrelated fatigue criterion. The contact fatigue life of clasp joint structure is analyzed by using finite element software based on S-N material fatigue curve and Miner linear cumulative damnification principle. The main conclusions are as follows.

1) Contact fatigue analysis shows that the fatigue life of clasp joint structure is four years. The most dangerous position of fatigue failure is the connector of buoy and clasp, which results from stress concentration.

2) Using the fatigue analysis function of ANSYS, the reliability of clasp joint structure is analyzed. The results show that the fatigue analysis function applied to mechanical structure is feasible, simple, economical and efficient, with a larger engineering application value.

3) The study only considers the impact of vertical vibration in the worst marine environment, without the factors of horizontal vibration, weld, and corrosion. Meanwhile, the marine environment is varied with the season. These factors referred above can be considered in the further study.

\section{References}

[1] Wang Qing-feng,He Li-dong. Analysis of fatigue life for contacting-type flexible seal on rotary air preheater based on ansys[J]. Thermal Power Generation, 2010, 39(1):32-35.(in Chinese)

[2] Wang Li-quan,Sun Zhi-juan,Meng Qing-xin. Finite element analysis for the contact problem of check ring joint structure in underwater skirt pile gripper[J]. Equipment Manufacturing Technology, 2006(4):9-11. (in Chinese)

[3] Liu Peng-fei,Zheng jin-yang,Sun guo-you. Fatigue lifetime evaluation of buried pipeling under internal pressure and vibrating loads[J]. Journal of Mechanical Strength, 2010, 32(1):125-129. (in Chinese)

[4] Liu Bao-xue. Fatigue reliability analysis of submarine pipe line under wave life force[J].Chemical Engineering \& Equipment,2008(9):89-94. (in Chinese)

[5] Xu Jin,Shi Zhao-dong,Zhang Kang. Morison equation based fatigue life analysis of submarine pipe line[J]. Journal of Chengde Petroleum College, 2009, 11(4):21-23. (in Chinese)

[6] Hu Rongcai,Xu Bingye. Study of a new model for prediction of contact fatigue life[J]. Engineer Mechanics, 1994, 11(3):47-54. (in Chinese)

[7] Han Dong,Liu Fu-shun,Li Yan,Xu Hui-bin. Simulation calculation of the tie-in clasp stress of the TiNiFe SMA tube[J]. Acta Aeronautica et Astronautica Sinica, 2006, 27(4):703-707. (in Chinese)

[8] Liu Yong-shou,Zhi You-hai,Liu Jun, Yue Zhu-feng. Fatigue life(S-N) Analysis of Ti-Ni pipe joint for the piping system[J]. Aircraft Design, 2009, 29(2):13-17. (in Chinese) 